\title{
Promotion de l'intendance en matière d'antimicrobiens : Résumé du rapport de 2015 du CDMTI
}

\author{
Khan $\mathrm{F}^{1}$, Arthur $\mathrm{J}^{1 *}$, Maidment $\mathrm{L}^{1}$, Blue $\mathrm{D}^{2}$ au nom du Comité directeur sur les maladies \\ transmissibles et infectieuses (CDMTI) de 2015 du groupe de travail chargé de l'intendance en \\ matière de l'utilisation d'antimicrobiens ${ }^{3}$
}

\section{Résumé}

Contexte : La résistance aux antimicrobiens est reconnue comme étant un important problème de santé publique à l'échelle mondiale qui a des répercussions transversales sur la santé humaine, la santé animale, I'alimentation, l'agriculture et l'environnement. Le Comité directeur sur les maladies transmissibles et infectieuses (CDMTI) du Réseau pancanadien de santé publique a créé un groupe de travail chargé de l'intendance en matière d'antimicrobiens afin d'examiner cette question d'un point de vue canadien.

Objectif : Résumer les principaux résultats du rapport du groupe de travail qui a permis de déterminer les éléments essentiels des programmes d'intendance en matière d'antimicrobiens, des pratiques exemplaires, des principaux défis, des lacunes et des recommandations pour promouvoir l'intendance à travers les juridictions.

Méthodologie : Des stratégies de recherche ont été élaborées pour identifier les ouvrages scientifiques, la littérature grise et les sites Web pertinents sur l'intendance en matière d'antimicrobiens. Les renseignements ont fait l'objet d'un examen et, en se fondant sur ces données probantes, des opinions d'experts et l'établissement d'un consensus, le groupe de travail a mis en évidence les éléments essentiels, les pratiques exemplaires, les principaux défis et les lacunes puis il a formulé des recommandations visant à promouvoir l'intendance en matière d'antimicrobiens au Canada.

Résultats : Les quatre éléments d'une initiative prometteuse portant sur l'intendance en matière d'antimicrobiens étaient les suivants : le leadership, les interventions, la surveillance et l'évaluation ainsi que les recherches futures. Les pratiques exemplaires comprennent une approche multisectorielle et multidimensionnelle nécessitant la participation d'un vaste éventail d'intervenants en matière d'organisation des soins de santé à l'échelle nationale, provinciale, territoriale et locale. Les principales lacunes et les principaux défis cernés étaient les suivants : la réussite et la durabilité des interventions liées à l'intendance nécessitent une expertise et des ressources appropriées et soutenues; il existe peu de données probantes sur la façon de mettre en œuvre efficacement les lignes directrices en matière de traitement; et l'assurance de l'accessibilité, de la normalisation et de l'uniformité de l'utilisation chez les professionnels de la santé pose problème.

Les recommandations formulées à l'intention du CDMTI sur la façon de promouvoir l'intendance en matière $d^{\prime}$ 'antimicrobiens à travers les juridictions étaient les suivantes : mettre en place une infrastructure nationale; mettre au point des pratiques exemplaires afin de mettre en œuvre les programmes d'intendance; faire progresser l'éducation et promouvoir la sensibilisation; mettre en place des lignes directrices, des ressources, de la formation et des outils uniformes fondés sur des données probantes; mandater l'intégration de l'éducation en matière d'intendance; mettre au point des outils de vérification et de rétroaction; établir des points de référence et des objectifs de rendement pour l'intendance; et mener des évaluations des programmes d'intendance en temps opportun.

Conclusion : Les conclusions du présent rapport permettront d'orienter une approche plus systématique à l'égard de l'intendance en matière d'antimicrobiens à l'échelle du Canada.

\section{Affiliations}

${ }^{1}$ Centre de la lutte contre les maladies transmissibles et les infections, Agence de la santé publique du Canada, Ottawa (Ontario)

${ }^{2}$ Bureau du médecin-hygiéniste en chef, Edmonton (Alberta)

${ }^{3}$ Voir la section Remerciements pour obtenir une liste complète des membres du groupe de travail

${ }^{*}$ Correspondance : jacqueline. arthur@phac-aspc.gc.ca
Citation proposée : Khan F, Arthur J, Maidment L, Blue D au nom du Comité directeur des maladies transmissibles et infectieuses (CDMTI) de 2015 du groupe de travail chargé de l'intendance en matière de I'utilisation d'antimicrobiens. Promotion de l'intendance en matière d'antimicrobiens : Résumé du rapport de 2015 du CDMTI. Relevé des maladies transmissibles au Canada. 2016;42(11):264-8. 


\section{Introduction}

La résistance aux antimicrobiens est reconnue comme un problème de santé mondial de plus en plus important qui menace la prévention, le contrôle et le traitement efficaces d'un large éventail d'infections. Au Canada, l'émergence d'organismes résistants aux antimicrobiens a été désignée comme une préoccupation importante dans les milieux de soins de santé et parmi les populations à risque. Étant donné que la résistance aux antimicrobiens peut apparaître chez les bactéries en réaction à une pression antimicrobienne sélective (c.-à-d. lorsque les bactéries sont en présence d'un médicament antimicrobien), il existe un risque que de moins en moins d'antimicrobiens demeurent efficaces à l'avenir. Il a été démontré que l'antibiothérapie inutile représente un lourd fardeau de l'utilisation inappropriée des antimicrobiens au Canada (1-4). En raison de préoccupations croissantes à propos du lien entre I'utilisation d'antimicrobiens et la résistance aux antimicrobiens, une réorientation vers une utilisation plus prudente des antimicrobiens a été l'un des domaines mis en évidence pour la lutte contre la propagation de la résistance aux antimicrobiens (5-8).

L'intendance en matière d'antimicrobiens est responsable de la planification et de la gestion des ressources afin de prévenir et de modérer l'évolution de la résistance aux antimicrobiens. Les initiatives en matière $d$ 'intendance et les programmes connexes abordent habituellement des enjeux liés à l'utilisation d'antimicrobiens afin de limiter la propagation de la résistance aux antimicrobiens et de préserver l'efficacité des antimicrobiens existants. L'intendance vise différents milieux et peut également englober des activités à l'extérieur du secteur de la santé humaine dans le cadre d'un modèle Une santé. Le rapport a reconnu que des mesures parallèles sont nécessaires en médecine vétérinaire, en alimentation animale et dans les secteurs de l'agriculture; cependant, il n'était axé que sur le secteur de la santé humaine.

Le Réseau pancanadien de santé publique (RSP) représente I'infrastructure nationale de santé publique du Canada qui permet de se pencher sur de tels problèmes de santé publique. En 2014-2015, le RSP a commencé à déterminer les composants d'un cadre de santé publique pancanadien sur la résistance aux antimicrobiens, en se concentrant sur les principaux éléments d'intendance qui concernent l'utilisation des antimicrobiens dans le secteur de la santé humaine. Le groupe de travail chargé de I'intendance en matière de l'utilisation d'antimicrobiens du Comité directeur sur les maladies transmissibles et infectieuses (CDMTI) a été mandaté par le CDMTI dans le cadre du RSP pour déterminer les éléments essentiels d'une approche d'intendance pancanadienne ainsi que les lacunes et les défis principaux et pour formuler des recommandations relatives à des façons de promouvoir l'intendance à travers les jurisdictions.

L'objectif du présent article est de résumer le Rapport du groupe de travail du CDMTI sur la gestion de I'utilisation d'antimicrobiens (9), qui détermine les éléments essentiels des programmes de gestion des antimicrobiens et des pratiques exemplaires dans les milieux de soins de santé humaine au Canada, met en évidence les défis et lacunes et présente une série de recommandations visant à promouvoir l'intendance en matière d'antimicrobiens au Canada.

\section{Méthodologie}

Le groupe de travail chargé de l'intendance en matière de I'utilisation d'antimicrobiens était composé d'experts en maladies infectieuses, de médecins de famille et d'autres médecins, d'épidémiologistes, de microbiologistes et de spécialistes de la santé publique. Des stratégies de recherche ont été élaborées pour identifier les ouvrages scientifiques, la littérature grise et les sites Web pertinents sur l'intendance en matière d'antimicrobiens; elles ont été complétées par des renseignements supplémentaires fournis par les membres. Les renseignements ont fait l'objet d'un examen et, en se fondant sur ces données probantes, des opinions d'experts et l'établissement d'un consensus, le groupe de travail a mis en évidence les éléments essentiels, les pratiques exemplaires, les principaux défis et les lacunes puis il a formulé des recommandations visant à promouvoir l'intendance en matière d'antimicrobiens au Canada.

\section{Résultats}

Les stratégies de recherche ont permis d'examiner et de résumer 400 articles aux fins de discussion au sein du groupe de travail. Aux fins du présent rapport, l'intendance en matière d'antimicrobiens a été définie comme des « interventions coordonnées visant à promouvoir, à améliorer, à surveiller et à évaluer I'utilisation judicieuse des antimicrobiens afin de préserver leur efficacité future, de protéger la santé humaine et d'en faire la promotion » $(9,10)$.

\section{Éléments essentiels}

Des programmes d'itendance prometteurs indiquent que de solides mesures interdisciplinaires de santé publique et un fort engagement politique peuvent entraîner une diminution mesurable de la résistance aux antimicrobiens et l'amélioration de l'utilisation optimale des antimicrobiens dans les milieux de soins de santé. Même si de plus amples recherches sont manifestement nécessaires pour valider cette hypothèse ainsi que des résultats connexes dans les milieux communautaires, quatre éléments essentiels des initiatives et des programmes $d$ 'intendance en matière d'antimicrobiens prometteurs sont ressortis : le leadership, les interventions, la surveillance et l'évaluation ainsi que la recherche (tableau 1).

\section{Tableau 1 : Description des quatre éléments essentiels de l'intendance en matière d'antimicrobiens}

\begin{tabular}{|l|l|}
\hline \multicolumn{1}{|c|}{$\begin{array}{c}\text { Élément } \\
\text { essentiel }\end{array}$} & \multicolumn{1}{c|}{ Description } \\
\hline Leadership & $\begin{array}{l}\text { Les interventions réussies liées à la gestion sont fondées } \\
\text { sur la responsabilisation, les ressources et l'expertise } \\
\text { appropriées et soutenues, le soutien adéquat ainsi que } \\
\text { la formation et elles comprennent la participation de } \\
\text { spécialistes de façon interdisciplinaire. }\end{array}$ \\
\hline Interventions & $\begin{array}{l}\text { Les interventions efficaces en matière d'intendance sont } \\
\text { complexes et multidimensionnelles. Elles consistent } \\
\text { en des services de sensibilisation, d'éducation et } \\
\text { d'orientation. En outre, elles comprennent des outils de } \\
\text { diagnostic ainsi que d'autres types d'outils qui fournissent } \\
\text { en temps opportun des renseignements fondés sur des } \\
\text { données probantes, et elles font participer de multiples } \\
\text { groupes cibles pour un effet maximal. }\end{array}$ \\
\hline $\begin{array}{l}\text { Surveillance et } \\
\text { évaluation }\end{array}$ & $\begin{array}{l}\text { Afin d'établir l'utilisation appropriée des antimicrobiens, } \\
\text { la littérature repère systématiquement le rôle essentiel } \\
\text { des points de référence, de la vérification et de } \\
\text { l'évaluation des systèmes. }\end{array}$ \\
\hline $\begin{array}{l}\text { Elle comprend le développement, l'application et la } \\
\text { mobilisation de connaissances. L'expertise provenant de } \\
\text { toutes les disciplines de recherche doit être mise à profit } \\
\text { afin de combler les lacunes en matière d'information } \\
\text { et de veiller à ce que les données probantes soient } \\
\text { disponibles et utilisées en vue de plus grandes } \\
\text { répercussions. }\end{array}$ \\
\hline
\end{tabular}




\section{Initiatives et pratiques exemplaires}

Le groupe de travail a identifié des programmes d'intendance efficaces qui avaient fait l'objet d'une évaluation à la fois au Canada et dans d'autres pays. Les initiatives canadiennes sont résumées dans le tableau 2 . Après l'examen de ces programmes, le groupe de travail a conclu que de solides mesures interdisciplinaires de santé publique et un fort engagement politique peuvent entraîner une diminution mesurable de la résistance aux antimicrobiens et l'amélioration de l'utilisation optimale des antimicrobiens dans les milieux de soins de santé.

Tableau 2 : Exemples de pratiques exemplaires reliées à l'intendance en matière d'antimicrobiens au Canada'

\begin{tabular}{|c|c|c|}
\hline $\begin{array}{l}\text { Nom et niveau de } \\
\text { l'intervention }\end{array}$ & $\begin{array}{l}\text { Description du } \\
\text { programme }\end{array}$ & Résultat \\
\hline $\begin{array}{l}\text { Alberta et } \\
\text { Colombie-Britannique } \\
\text { Des pilules contre } \\
\text { tous les microbes? } \\
\text { (Instaurée à Grande } \\
\text { Prairie, Alberta) (7) }\end{array}$ & $\begin{array}{l}\text { Le programme était } \\
\text { axé sur deux éléments: } \\
\text { fournir des ressources } \\
\text { éducatives aux } \\
\text { médecins, aux } \\
\text { infirmières et } \\
\text { aux pharmaciens } \\
\text { dans les hôpitaux } \\
\text { communautaires et } \\
\text { les établissements de } \\
\text { soins de longue durée; } \\
\text { fournir une éducation } \\
\text { à propos des risques } \\
\text { de la résistance aux } \\
\text { antimicrobiens et } \\
\text { de l'utilisation des } \\
\text { antimicrobiens à } \\
\text { l'intention du grand } \\
\text { public. }\end{array}$ & $\begin{array}{l}\text { Une approche } \\
\text { multimédia } \\
\text { comprenant des } \\
\text { documents imprimés, } \\
\text { des campagnes } \\
\text { publicitaires ainsi que } \\
\text { de la formation et } \\
\text { de la sensibilisation } \\
\text { continues à l'intention } \\
\text { de personnes de tous } \\
\text { les groupes d'âge } \\
\text { et d'une variété de } \\
\text { professionnels de la } \\
\text { santé s'est avérée } \\
\text { toucher de nombreux } \\
\text { publics cibles. }\end{array}$ \\
\hline $\begin{array}{l}\text { Québec } \\
\text { Stratégie éducative } \\
\text { multidimensionnelle } \\
\text { sur la prescription } \\
\text { d'antibiotiques }\end{array}$ & $\begin{array}{l}\text { Les lignes directrices } \\
\text { ont été diffusées à } \\
\text { l'aide d'une stratégie } \\
\text { multidisciplinaire } \\
\text { et principalement } \\
\text { en ligne, y compris } \\
\text { des versions } \\
\text { téléchargeables sur } \\
\text { un site Web dédié et } \\
\text { de la promotion par } \\
\text { les professionnels } \\
\text { et les experts lors } \\
\text { d'événements } \\
\text { éducatifs. Elles } \\
\text { ont également été } \\
\text { fortement acceptées } \\
\text { par l'industrie } \\
\text { pharmaceutique. }\end{array}$ & $\begin{array}{l}\text { Des lignes directrices } \\
\text { conviviales fondées } \\
\text { sur des données } \\
\text { scientifiques, élaborées } \\
\text { par des organismes } \\
\text { crédibles, appuyées } \\
\text { par des organisations } \\
\text { professionnelles et } \\
\text { faisant l'objet d'une } \\
\text { promotion active } \\
\text { ont une incidence } \\
\text { considérable sur la } \\
\text { réduction des pratiques } \\
\text { de prescription } \\
\text { inappropriée } \\
\text { d'antimicrobiens au sein } \\
\text { de la collectivité. }\end{array}$ \\
\hline $\begin{array}{l}\text { Ontario } \\
\text { Traitement des } \\
\text { infections respiratoires } \\
\text { dans la communauté }\end{array}$ & $\begin{array}{l}\text { Une stratégie éducative } \\
\text { multidisciplinaire } \\
\text { à l'échelle de la } \\
\text { collectivité a été } \\
\text { utilisée en Ontario, } \\
\text { dans le but d'améliorer } \\
\text { l'adoption des lignes } \\
\text { directrices cliniques } \\
\text { ainsi que l'utilisation } \\
\text { des antimicrobiens. }\end{array}$ & $\begin{array}{l}\text { Les éléments de la } \\
\text { réussite étaient la } \\
\text { création de documents } \\
\text { crédibles et conviviaux, } \\
\text { l'éducation du public, } \\
\text { des pharmaciens et } \\
\text { des cliniciens et le } \\
\text { soutien apporté aux } \\
\text { professionnels de } \\
\text { la santé motivés à } \\
\text { l'échelle locale dans } \\
\text { la coordination des } \\
\text { éléments éducatifs. }\end{array}$ \\
\hline
\end{tabular}

Tableau 2 suite

\begin{tabular}{|l|l|l|}
\hline \hline Ontario & Les principales & Les principaux \\
Réduction de C. difficile & techniques & facteurs de succès \\
dans les unités de soins & d'intervention & étaient les ressources \\
intensifs (lancée au & étaient le recours & humaines appropriées \\
Mount Sinai Hospital, & à un médecin ou & pour une direction \\
Toronto [Ontario]) & à pharmacien & efficace, le soutien à \\
spécialisé en maladies & la prise de décisions, \\
& infectieuses, la & la vérification et \\
& distribution de & la rétroaction \\
& documents éducatifs & prospectives, ainsi \\
& pertinents aux & que l'échange de \\
professionnels de & connaissances par \\
& la santé en milieu & l'entremise de la \\
& communication entre \\
& hospitalier, puis & pairs. \\
& une vérification et & \\
& une rétroaction & \\
& prospectives dans & \\
& les unités de soins & \\
& intensifs. & \\
\hline
\end{tabular}

Ce tableau ne présente qu'une partie des programmes au Canada. Pour une liste plus complète des initiatives, au Canada comme à l'étranger, veuillez consulter le rapport complet (9)

\section{Principaux défis et lacunes en matière de connaissances}

Après la détermination des éléments essentiels d'une intendance efficace et l'examen des initiatives et des programmes d'intendance réussis, d'importants défis et des lacunes en matière de connaissances se sont dégagés de la discussion au sein du groupe de travail (tableau 3). Par exemple, le succès et la durabilité des interventions liées à l'intendance nécessitent une expertise et des ressources appropriées et durables (ce qui n'est pas toujours possible dans une région donnée ou un milieu donné) et il existe des lacunes à propos de l'orientation en matière de traitement, de ses avantages, de la façon de le mettre en œuvre, ainsi que d'assurer l'accessibilité, la normalisation et l'uniformité de son utilisation parmi les professionnels de la santé.

\section{Tableau 3 : Lacunes et défis actuels reliés à l'intendance} en matière d'antimicrobiens

\section{Défis}

Ressourcement approprié : Le succès et la durabilité des interventions liées à l'intendance nécessitent des ressources appropriées et durables, en particulier, une expertise appropriée (ce qui n'est pas toujours possible dans une région donnée ou un milieu donné).

Accès et uniformité des lignes directrices : Les médecins canadiens ont déclaré avoir de la difficulté à localiser des ressources pertinentes au sujet de la résistance aux antimicrobiens en général et des protocoles d'analyse. Différents professionnels prescripteurs ont accès à différents documents en matière de lignes directrices.

Suivi de l'efficacité des traitements et des programmes : Intégration des tests de contrôle post-traitement (remise en culture au site d'infection afin de déterminer si l'infection est toujours présente) dans les documents d'orientation. Il est difficile de déterminer l'efficacité des programmes et des campagnes en raison du manque d'indicateurs normalisés.

\section{Lacunes}

Manque de formation : Des initiatives d'éducation et de formation en matière d'intendance à l'intention de tous les professionnels prescripteurs sont nécessaires.

Détermination des situations où il convient de ne pas prescrire : De plus amples recherches sont nécessaires pour déterminer si la production d'outils d'orientation à l'intention des prescripteurs à propos des situations où il convient de ne pas prescrire des antibiotiques serait avantageuse et la façon de les mettre en œuvre.

Nécessité d'un diagnostic rapide : Des outils de diagnostic rapide dans les points de service qui permettent de distinguer les infections virales des infections bactériennes ainsi que de déterminer et de caractériser les bactéries résistantes sont nécessaires pour orienter l'utilisation appropriée des antimicrobiens. 


\section{Recommandations}

En se fondant sur les éléments essentiels, les pratiques exemplaires, les lacunes et les défis actuels, le groupe de travail a mis au point des recommandations pour promouvoir I'intendance en matière d'antimicrobiens au Canada (tableau 4). La mise en œuvre des recommandations du rapport doit prendre en compte les avancées actuelles dans les domaines de recherche des politiques et des programmes.

\section{Tableau 4 : Recommandations au CDMTI relatives aux éléments essentiels des pratiques reliées à l'intendance en matière d'antimicrobiens}

\begin{tabular}{|c|c|}
\hline $\begin{array}{l}\text { Élément } \\
\text { essentiel }\end{array}$ & Recommandation(s) au CDMTI \\
\hline Leadership & $\begin{array}{l}\text { Mettre en place une infrastructure nationale (p. ex. } \\
\text { gouvernance, réseau, ressources, etc.) pour soutenir } \\
\text { les provinces et les territoires dans l'élaboration de } \\
\text { programmes d'intendance dans les milieux de soins de } \\
\text { santé ainsi que communautaires. }\end{array}$ \\
\hline \multirow{3}{*}{ Interventions } & $\begin{array}{l}\text { Des pratiques exemplaires, des points de repère et } \\
\text { des normes en matière d'activités d'éducation et } \\
\text { de sensibilisation qui nécessitent l'engagement de } \\
\text { différents groupes de prescripteurs et qui sont axés à } \\
\text { la fois sur les prescripteurs et les utilisateurs. }\end{array}$ \\
\hline & $\begin{array}{l}\text { Les lignes directrices, les renseignements, les outils } \\
\text { et la formation à l'intention des professionnels } \\
\text { prescripteurs et des utilisateurs doivent être } \\
\text { complets, accessibles et uniformes afin de soutenir les } \\
\text { prescripteurs locaux. }\end{array}$ \\
\hline & $\begin{array}{l}\text { Les universités, les collèges et les écoles techniques } \\
\text { qui forment les futurs prescripteurs devraient intégrer } \\
\text { de l'éducation et de la formation continue obligatoires } \\
\text { en matière de gestion à l'intention des professionnels } \\
\text { prescripteurs. }\end{array}$ \\
\hline \multirow[b]{2}{*}{$\begin{array}{l}\text { Surveillance } \\
\text { et évaluation }\end{array}$} & $\begin{array}{l}\text { Des outils de vérification et de rétroaction fondés sur } \\
\text { des données probantes devraient être élaborés pour } \\
\text { soutenir les prescripteurs, et les lignes directrices à } \\
\text { l'intention des prescripteurs devraient être évaluées, } \\
\text { adaptées et mises en œuvre à l'échelle régionale et à } \\
\text { l'échelle locale. }\end{array}$ \\
\hline & $\begin{array}{l}\text { Des points de repère devraient être établis en matière } \\
\text { d'utilisation optimale des antimicrobiens par type } \\
\text { d'infection ainsi que pour les populations les plus } \\
\text { vulnérables à l'infection, les jurisdictions devraient } \\
\text { travailler ensemble pour établir des objectifs de } \\
\text { rendement en matière d'intendance dans les hôpitaux } \\
\text { et dans d'autres milieux, et des évaluations des } \\
\text { programmes d'intendance devraient être réalisées en } \\
\text { temps opportun et rendues accessibles au public. }\end{array}$ \\
\hline \multirow{2}{*}{$\begin{array}{l}\text { Recherche } \\
\text { future }\end{array}$} & $\begin{array}{l}\text { Davantage de données probantes sur les } \\
\text { comportements des professionnels prescripteurs } \\
\text { devraient être recueillies, partagées et mobilisées afin } \\
\text { que des interventions particulières pour ces professions } \\
\text { puissent être mises en œuvre. }\end{array}$ \\
\hline & $\begin{array}{l}\text { Des modifications devraient être apportées aux } \\
\text { règlements sur les pratiques des professionnels } \\
\text { de la santé et de plus amples données probantes } \\
\text { devraient être recueillies pour orienter et appuyer ces } \\
\text { modifications. }\end{array}$ \\
\hline
\end{tabular}

Le groupe de travail a également relevé deux autres facteurs à prendre en considération. Tout d'abord, que la sécurité des patients, l'évitement des effets indésirables et des pratiques efficaces de prévention et de contrôle des infections sont tous des facteurs importants qui contribuent également aux initiatives d'intendance en matière d'antimicrobiens. Enfin, le groupe de travail indique que l'évaluation des programmes et des initiatives d'intendance devrait faire l'objet d'une promotion auprès des organismes d'octroi, et qu'on devrait envisager son inclusion dans les priorités face à l'utilisation et à la résistance et aux antimicrobiens en matière de financement.

\section{Conclusion}

Le groupe de travail a déterminé que le leadership, les interventions appropriées, la surveillance et l'évaluation, la nécessité d'acquérir davantage de connaissances sur I'intendance efficace-ainsi que les données probantes permettant sa promotion-sont des éléments essentiels des initiatives et des programmes prometteurs d'intendance en matière d'antimicrobiens. Il existe un certain nombre de défis importants et de lacunes en matière de connaissances qui peuvent être résolus par les recommandations formulées par le groupe de travail. Elles font actuellement l'objet d'un examen par les gouvernements et les experts en soins de santé.

Une meilleure gestion de l'utilisation des antimicrobiens est une responsabilité partagée entre différents intervenants, notamment les professionnels de santé publique et de soins de santé, les organisations de soins de santé, les gouvernements locaux, provinciaux, territoriaux et nationaux, le milieu de la recherche ainsi que d'autres entités qui fournissent et utilisent des antimicrobiens et qui fournissent des soins aux personnes qui les utilisent. L'élaboration, la promotion et la mise en œuvre d'initiatives visant à promouvoir l'utilisation optimale des antimicrobiens à l'échelle du Canada nécessiteront la collaboration entre tous ces intervenants, afin de faciliter la création d'une synergie pour lutter efficacement contre la résistance aux antimicrobiens.

\section{Remerciements}

Les auteurs souhaitent remercier les membres du groupe de travail chargé de l'intendance en matière de l'utilisation d'antimicrobiens de 2015 du CDMTI pour leur contribution et leurs conseils d'expert :

Dean Blue (coprésident), bureau du médecin hygiéniste en chef (Alberta); John Conly, Services de santé de l'Alberta et Université de Calgary; Gary Garber, Santé publique Ontario; Greg German, ministère de la Santé de l'île-du-Prince-Édouard; Melissa Helferty, ministère de la Santé et des Soins de longue durée de l'Ontario; David Patrick, Centre de contrôle des maladies de la Colombie-Britannique; Jeff Powis, Hôpital Michael Garron, Toronto (Ontario); Richard Rusk, Santé Manitoba, Vie saine et Aînés; Nadine Sicard, ministère de la Santé et des Services sociaux (Québec)

Agence de la santé publique du Canada : Kanchana Amaratunga, Jacqueline Arthur (Secrétariat), Christine Cryan (Secrétariat), Monika Dankova, Rita Finley, Katie Freer (jusqu'en août 2015), 
Robert Gervais, Edward Gertler, Leanne Maidment (coprésidente), Stephanie Mehta (Secrétariat), Valerie Sarazin (Secrétariat, jusqu'en juillet 2015), Shari Silber (Secrétariat), Christine N. Soon, Deepika Sriram (Secrétariat, jusqu'en octobre 2015), Geneviève Tremblay (jusqu'en août 2015)

\section{Conflit d'intérêts}

Aucun. Jacqueline Arthur est l'une des rédactrices invitées pour la présente publication et se récuse des décisions de la rédaction relatives au présent article. Les décisions de la rédaction ont été prises par la rédactrice en chef, $D^{\text {re }}$ Patricia Huston, et l'autre rédactrice invitée, $D^{r e}$ Kanchana Amaratunga.

\section{Financement}

Ce travail a été appuyé par l'Agence de la santé publique du Canada.

\section{Références}

1. Wang E, Einarson T, Kellner J, Conly J. Antibiotic prescribing patterns for respiratory tract infections in preschool children in Saskatchewan: Evidence for overprescribing for viral syndromes. Clin Infect Dis. 1999;29:155-60.

2. Nicolle LE. Infection prevention issues in long-term care. Curr Opin Infect Dis. 2014;27(4):363-69.

3. Silver SA, Baillie L, Simor AE. Positive urine cultures: A major cause of inappropriate antimicrobial use in hospitals? Can J Infect Dis Med Microbiol. 2009;20(4):107-11.

4. Leis JA, Rebick GW, Daneman N, Wayne GL, Poutanen SM, Lo P, Larocque M, Shojania KG, McGeer A. Reducing antimicrobial therapy for asymptomatic bacteriuria among noncatheterized inpatients: A proof-of-concept study. Clin Infec Dis. 2014;58(7)980-3.
5. Moody J, Cosgrove SE, Olmsted RN, Septimus E, Aureden K, Oriola S. et al, Antimicrobial stewardship: A collaborative partnership between infection preventionists and health care epidemiologists. Am J Infect Control. 2012;40:94-5.

6. McKay RM, Vrbova L, Fuertes E, Chong M, David S, Dreher $\mathrm{K}$ et al. Evaluation of the Do Bugs Need Drugs Program in British Columbia: Can we curb antibiotic prescribing? Can J Infect Dis Med Microbiol. 2011;22(1):19-24.

7. Carson M, Patrick DM. « Des pilules contre tous les microbes? „Programme d'education communautaire pour une utilisation judicieuse des antibiotiques. Relevé des maladies transmissibles au Canada. 2015;41(Suppl 4):5-9. Disponible à l'adresse : http://www.phac-aspc.gc.ca/ publicat/ccdr-rmtc/15vol41/dr-rm41s-4/overview-apercu_01fra.php.

8. British Columbia Centre for Disease Control [Internet]. Do Bugs Need Drugs? Annual evaluation report 2013/14. Vancouver BC: BCCDC; 2014. Disponible à l'adresse : http:// www.bccdc.ca/NR/rdonlyres/2CE3760F-4A41-4F7E-89DC2F8CD39A6F92/0/201314DBNDAnnualEvaluationRepo rt_FINAL_amended.pdf.

9. Réseau pancanadien de santé publique, Groupe de travail sur la gestion de l'utilisation d'antimicrobiens du Comité directeur sur les maladies transmissibles et infectieuses. [Internet]. Rapport final au Conseil du Réseau de santé publique : Gestion des antimicrobiens. Ottawa ON: RSPC; 2016 avr. Disponible à l'adresse : https://www.phn-rsp.ca/fr/ rapports-publications/gestion-antimicrobiens.html.

10. National Institute for Health and Care Excellence (NICE) [Internet]. UK Committee Task Group on Antimicrobial Use Stewardship. Antimicrobial Stewardship: Systems and Processes for Effective Antimicrobial Medicine Use. [mis à jour le 18 août 2015, consulté le 10 oct 2016]. Disponible à l'adresse : https://www.nice.org.uk/guidance/ng15. 